\title{
Traitement de la diarrhée aiguë et malnutrition de l'enfant
}

La diarrhée aiguë est une cause majeure de malnutrition chez l'enfant. Les traitements modernes de la diarrhée, fondés sur des concepts physiopathologiques, sont un moyen efficace de lutte contre la malnutrition dans le monde.

\section{Jehan-François Desjeux} Directeur de l'unité de recherches sur le diabète et la nutrition chez l'enfant (Inserm U83). a malnutrition de l'enfant est souvent imputée à l'injustice sociale et à la fatalité. Elle peut être envisagée également comme la conséquence d'un certain nombre de facteurs mieux identifiables, tels que l'insuffisance ou l'utilisation incomplète des aliments pris par l'enfant (figure $I$ ). Plus précisément, l'insuffisance alimentaire peut être elle-même liée à l'insuffisance de l'allaitement maternel, à une mauvaise répartition des aliments et à la quantité d'aliments disponibles pour la famille. D'un autre côté, l'utilisation ou le rendement nutritionnel chez l'enfant est fortement lié à son état de santé. Toutes les interventions sur l'éducation, l'environnement et le traitement des maladies devraient donc parvenir à améliorer l'état nutritionnel, et ceci d'autant plus que l'apport alimentaire est limité. A cet égard, les stratégies mises en place ces dernières années pour lutter contre les diarrhées aiguës du jeune enfant semblent exemplaires.

Cet article présente à grands traits les aspects scientifiques à l'origine de ces stratégies conçues essentiellement à partir de constatations épidémiologiques, économiques et physiologiques. La stratégie la mieux structurée est celle de l'Organisation Mondiale de la Santé. Ainsi, en principe, la volonté politique de réduire dans un pays ou une région les conséquences de la diarrhée peut s'appuyer sur une attitude scientifique [I].

\section{Épidémiologie et clinique}

Ce n'est que très récemment qu'ont été reconnues certaines données importantes concernant les maladies diarrhéiques aiguës chez les enfants de moins de 5 ans dans les pays en développement : elles représentent les premières causes de décès dans le monde, elles tendent à prendre la première place dans la liste des causes de morbidité, elles sont un facteur essentiel dans le déclenchement, la poursuite et l'aggravation de la malnutrition; enfin, elles sont à l'origine de la majorité des consultations hospitalières et des hospitalisations.

Sans entrer dans les détails méthodologiques, on peut concevoir que la cause d'un décès est souvent complexe, et, que même dans les meilleures conditions, il est souvent difficile de bien la préciser. Par exem- 


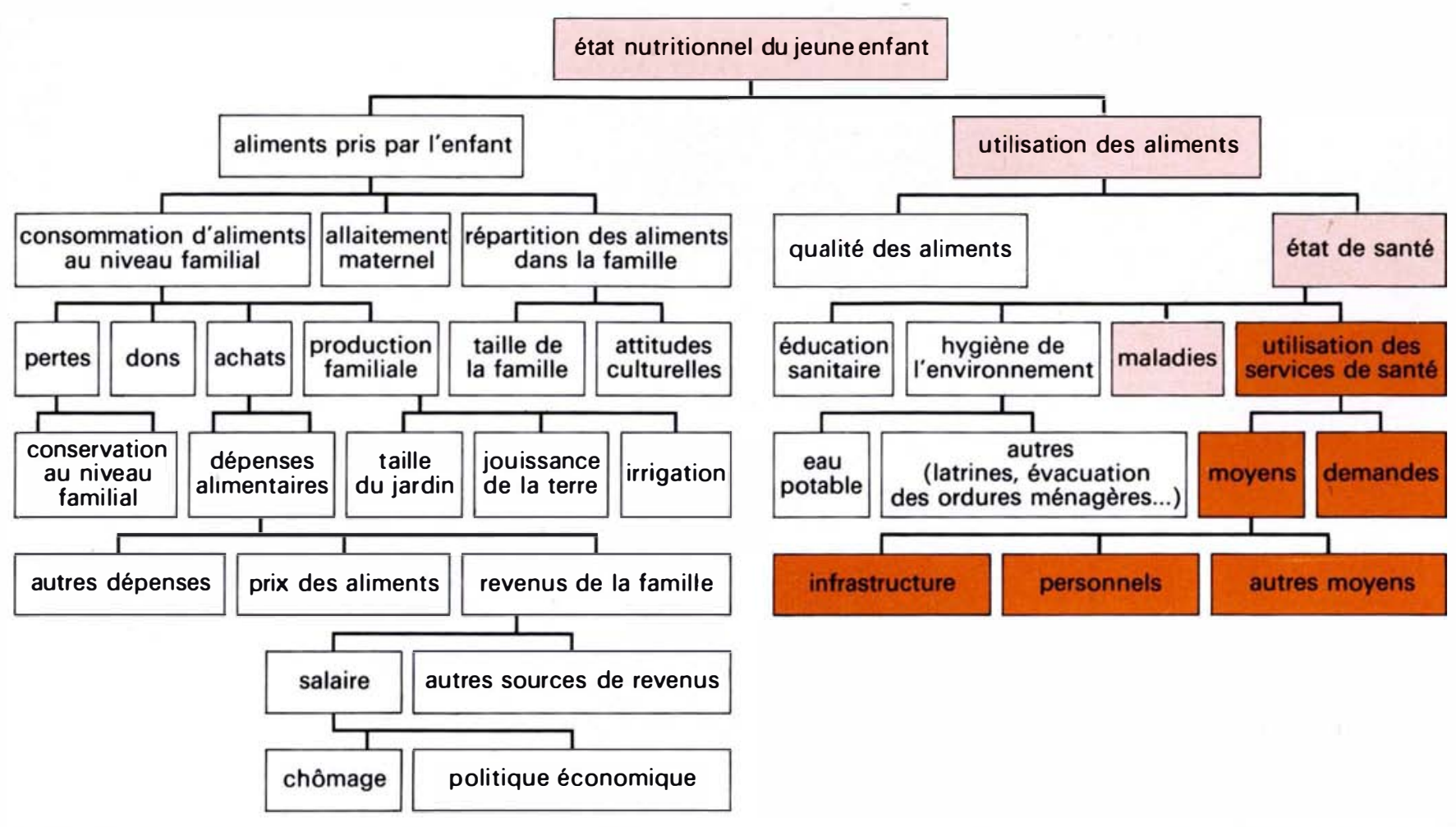

Figure I. Exemple d'un modèle causal hypothétique. Le rôle des diarrhées aiguës infectieuses sur l'état nutritionnel de l'enfant est représenté en rose; le rôle des systèmes de santé est représenté en rouge. (d'après Beghin J, Cap M, et Dujardin B. "Guide de l'évaluation nutritionnelle ". Document en préparation. OMS, Genève).

ple, un enfant malnutri (cause favorisante), atteint de rougeole (cause principale), décède à l'occasion d'une déshydratation par diarrhée (cause immédiate). Dès lors, si comme c'est l'habitude dans ce cas, seule la cause principale est déclarée, les autres causes sont masquées; dans l'exemple présent, il en sera ainsi de la malnutrition et de la diarrhée [I]. Il a donc été nécessaire de développer une méthodologie d'enquête sur le terrain. Ainsi, malgré l'imprécision des estimations de mortalité et de morbidité par diarrhée, les dernières évaluations à l'échelle mondiale sont saisissantes [2]. Tout d'abord, la population d'enfants de moins de 5 ans exposés à la maladie est de l'ordre de 338 millions, soit 75 millions en Afrique, 209 millions en Asie (sans m/s $n^{0} 7$ novembre 85 compter la Chine) et 54 millions en Amérique Latine. Or, chacun de ces enfants présente en moyenne plus de deux épisodes de diarrhée chaque année; cette fréquence est presque doublée entre 6 et i I mois de vie. Il y a donc chaque année entre 750 millions et I milliard d'épisodes de diarrhée dans cette tranche d'âge. Tous les épisodes n'ont pas la même gravité mais environ $0,5 \%$ d'entre eux sont mortels, ce qui signifie que chaque année il y aurait de 3,8 à 5 millions d'enfants qui meurent de diarrhée. En d'autres termes, la diarrhée représente environ un tiers des causes de décès dans les 5 premières années. Ces chiffres donnent une idée de l'ampleur de la tâche médicale à accomplir. Ils doivent cependant être précisés dans chaque région d'interven- tion. Ainsi, dans la plupart des pays d'Afrique, plus de $50 \%$ des décès des enfants de moins de 5 ans sont dus ou associés aux maladies diarrhéiques, les résultats variant selon les pays, les zones d'enquête, les saisons. A titre d'exemple, voici quelques résultats d'une enquête dans la région soudano-sahélienne du Sénégal : la prévalence instantanée de la diarrhée est considérable : dans une enquête portant sur I 854 enfants, I 75 enfants avaient une diarrhée le jour même de l'enquête et 264 d'entre eux avaient eu une diarrhée au cours de la semaine. Ainsi, chaque enfant de cette zone aurait chaque année plus de sept épisodes de diarrhée, d'une durée moyenne de 4,6 jours, soit plus d'un mois de diarrhée par an, en moyenne! Les garçons sont plus 


\section{REFERENCES}

I. Desjeux JF, Ducluzeau R, eds. La Diarrhée du Jeune. Paris : Editions Inserm, 1984.

2. Snyder JD, Merson MH. The magnitude of the global problem of acute diarrheal disease: a review of active surveillance data. Bull $W H O$ 1982; 60: 605-13.

3. Martorell R, Habicht JP, Yarbrough C, Lechtig RE, Klen R, Western KA. Acute morbidity and physical growth in Guatemalan children. Am $\mathcal{J}$ Dis Child 1975; 129 : $1296-9$.

4. Programme de lutte contre les maladies diarrhéiques. Le traitement des diarrhées aiguës à l'usage des médecins et des autres personnels de santé de niveau supérieur. Genève. Organisation Mondiale de la Santé, WHO/CDD/SER/80.2 Rev 1 1984: 27.

5. Scrimshaw NS. Significance of the interactions of nutrition and infection in children. In: Susskind RM, cd. Textbook of pediatric nutrition. New York: Raven Press, 1981: 229-40.

6. Ismail S, Swaby D, Macfarlane D, King D, de Benoist B. Relations entre diarrhée à rotavirus et contenu énergétique de l'alimentation dans un groupe d'enfants jamaicains d'âge préscolaire. Une étude prospective. In: Desjeux JF, Ducluzeau R, cds. La Diarrhée du Jeune. Paris: Editions Inserm, 1984: 53-6o.

7. Sinatra FR, Merrit RJ. Iatrogenic kwashiorkor in infants. Am 7 Dis Child 1981; 135: 21-3.

8. Société Française de Pédiatrie. Comité de Nutrition. Traitement diététique des diarrhées aiguës de l'enfant. Arch Fr Pediatr 1984; 4I : $211-6$.

9. American Academy of Pediatrics. Committee on nutrition. Use of oral fluid therapy and posttreatment feeding in a developed country.

touchés que les filles et il existe une protection relative pendant les six premiers mois, peut-être en rapport avec la pratique de l'allaitement maternel dans cette région [I].

C'est un fait maintenant bien établi que la maladie diarrhéique plus que toute autre maladie infectieuse retentit sur la courbe de croissance pondérale et staturale du nourrison et de l'enfant [3]. Cet effet nutritionnel est modulé par d'autres facteurs comme l'insuffisance alimentaire, le sexe, la parité, le niveau socio-économique et la période de l'année pour la naissance et le sevrage. En effet, quand la diarrhée survient, toutes les conditions sont réunies pour favoriser la malnutrition: il se produit simultanément une augmentation des pertes digestives et des besoins énergétiques, et une diminution des entrées nutritionnelles. Les pertes digestives font partie de la définition même de la diarrhée. La perte en eau et en électrolytes se traduit cliniquement par une déshydratation aiguë dont l'appréciation réalisable par des moyens simples conditionne la décision thérapeutique initiale [4]. Ainsi, en plus des pertes hydroélectrolytiques, la diarrhée est responsable de pertes énergétiques par trois mécanismes :

(a) Les diarrhées aiguës infectieuses provoquent des pertes énergétiques sous forme de protides, de lipides et de glucides par vomissement et par un mécanisme de malabsorption et d'entéropathie exsudative. Elles entraînent également une malabsorption des vitamines $\mathrm{A}, \mathrm{B}_{1} \mathrm{2}$ et des folates. Les pertes caloriques peuvent être considérables et la durée de récupération peut s'étendre sur plusieurs semaines, même pour un bref épisode de diarrhée [5]. Cependant, la malabsorption semble plus importante et plus durable pour les infections à rotavirus que pour celles à $\mathrm{E}$. Coli

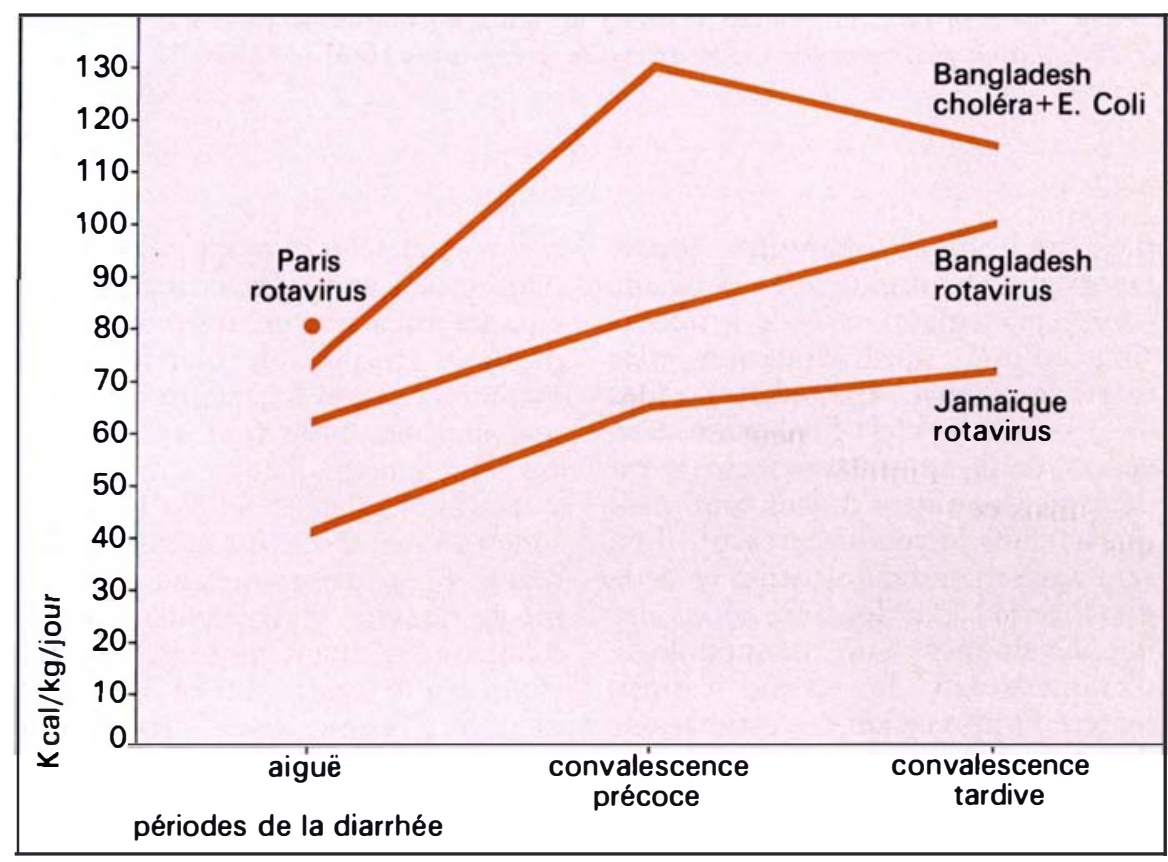

Figure 2. Consommation d'énergie pendant les périodes aiguës de convalescence précoce et de convalescence tardive des diarrhées infectieuses dans différentes régions [6]. 
entérotoxinogènes ou à Shigelles, même chez l'enfant bien nourri, réhydraté et réalimenté très rapidement $[\mathrm{I}]$, à plus forte raison chez l'enfant malnutri.

(b) De plus, la malnutrition est aggravée par la diminution des entrées énergétiques. Des études récentes ont mis en évidence une anorexie pendant l'épisode de diarrhée. Il existe des variations d'une étude à l'autre; ainsi dans le cas d'une diarrhée aiguë à rotavirus, un nourrisson consomme en moyenne $40 \mathrm{Kcal} / \mathrm{kg} /$ jour à la Jamaïque, 6o au Bangladesh et 80 à Paris [I]. La réalité du déficit est plus nette lorsque la consommation alimentaire est analysée pendant la période de convalescence $(f i-$ gure 2): ainsi, la consommation d'énergie s'élève graduellement de la phase aiguë à la phase de convalescence précoce et à la phase de convalescence tardive [6]. Cette anorexie est bien liée à l'infection intestinale car elle peut être reproduite expérimentalement chez l'animal. Certains facteurs aggravent probablement cette anorexie, comme l'environnement familial, l'agent infectieux de la diarrhée, l'état nutritionnel antérieur, la prise de certains médicaments et l'absence d'allaitement maternel (tableau I). En effet, la diminution de la fréquence de l'allaitement maternel s'associe vraisemblablement à l'apparition de la diarrhée à un âge plus jeune avec un retentissement nutritionnel plus important. La carence est encore aggravée par la restriction alimentaire encore trop souvent considérée comme nécessaire à la guérison. A l'origine de cette attitude, il y a peut-être le souci d'éviter l'intolérance alimentaire; mais cette mesure est d'autant plus néfaste que les réserves énergétiques de l'enfant sont plus faibles, c'est-à-dire qu'il est plus jeune ou déjà malnutri [7]. Au risque de malnutrition lié à cette coutume tenace et qui persiste malgré les recommandations $[4,8,9]$, il s'ajoute maintenant le risque lié à l'utilisation inappropriée des solutions de réhydratation.

(c) Le troisième élément qui favorise la malnutrition est l'altération du métabolisme énergétique, essentiellement en raison de la fièvre qui

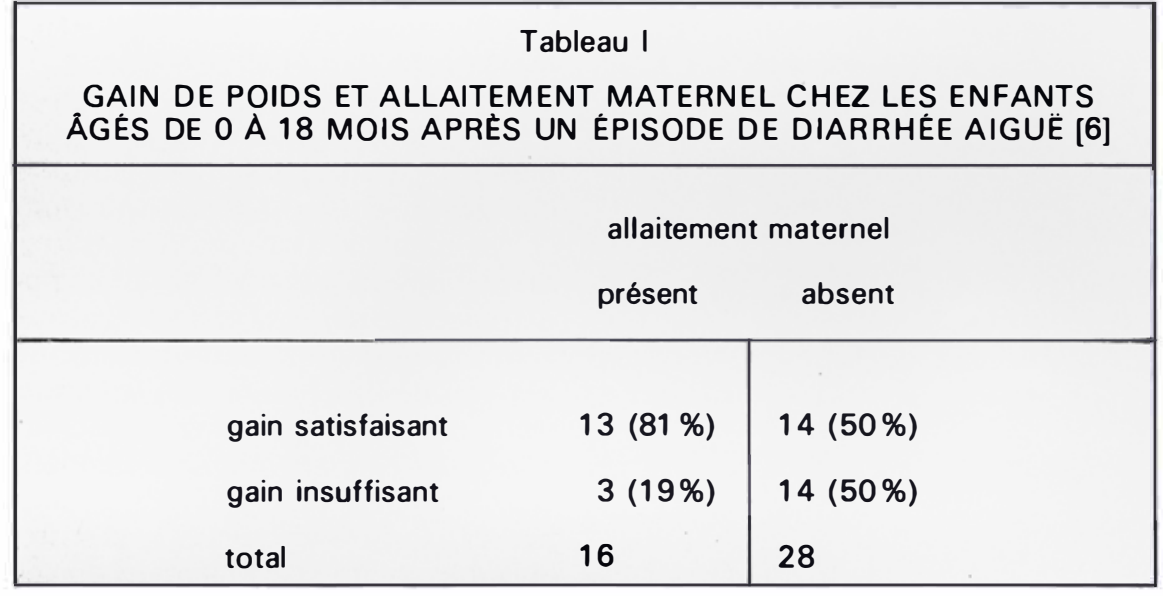

Tableau II

CARACTÉRISTIQUES CLINIQUES INITIALES DE 2 GROUPES D'ENFANTS DONT L'ÉVOLUTION AU $4^{\circ}$ JOUR S'EST FAITE VERS UN GAIN OU UNE PERTE DE POIDS [1]

\begin{tabular}{|l|c|c|}
\hline \multicolumn{1}{|c|}{ caractères cliniques } & prise de poids & perte de poids \\
variation du poids sur 4 jours & $+3,6 \%$ & $-2,3 \%^{*}$ \\
nombre d'enfants & 49 & 13 \\
filles & $51 \%$ & $49 \%$ \\
nombre de selles par jour & 4,6 & 4,9 \\
vomissements & $51 \%$ & $38 \%$ \\
signes cliniques de déshydratation & $33 \%$ & $38 \%$ \\
signes cliniques associés & $45 \%$ & $46 \%$ \\
température $38^{\circ}$ & $33 \%$ & $61 \% *$ \\
rotavirus dans les selles & $28 \%$ & $30 \%$ \\
\hline
\end{tabular}

* Différence significative entre les 2 groupes $(\mathrm{P}<0,05)$.

modifie le métabolisme de base. Ainsi, selon une étude portant sur les effets de la réalimentation précoce, la fièvre est le seul facteur associé à la perte de poids, malgré des apports hydriques et caloriques identiques dans les deux groupes (tableau II).

En conclusion, cette analyse un peu schématique et sûrement incomplète permet d'identifier la diarrhée aiguë infectieuse comme une cause fréquente et certaine de la malnutrition de l'enfant. Il est donc légitime de penser que le traitement de la diarrhée aiguë doit avoir un effet bénéfique sur l'état nutritionnel de l'enfant. Mais, un traitement efficace est-il envisageable dans un environnement très peu médicalisé?

\section{Physiopathologie des diarrhées aiguës}

Il est bien évident que le traitement des diarrhées aiguës remonte à la nuit des temps. Cependant, ce n'est que depuis vingt-cinq ans approximativement que ce traitement s'est révélé véritablement efficace. Ceci est dû en grande partie au développement des techniques de recherche 


\section{RÉFÉRENCES}

10. Katchalsky A, Curran PF. Nonequilibrium thermodynamics in biophysics. Cambridge: Harvard University Press, 1967; 248.

I I. Curran PF, Solomon AK. Ion and water fluxes in the ileum of rats. J Gen Physiol 1957; 41 : I 43-68.

12. Crane RK. Hypothesis for mechanism of intestinal active transport of sugars. Fed Proc 1962; $21: 891$

I 3. Finkelstein RA, LoSpalluto JJ. Production of highly purified choleragen and choleragenoid. $\mathcal{J}$ Infect Dis 1970; 121 (suppl): S63-72.

14. Desjeux JF, Tannenbaum C, Tai YH, Curran PF. Effects of sugar and amino acids on sodium movements across small intestine. Am 7 Dis Child 1973; 131 : 331-40.

15. Nalin DR, Cash RA, Islam R. Oral maintenance therapy for cholera in adults. Lancet 1962 ii : $370-73$.

16. Phillips RA. Water and electrolyte losses in cholera. Fed Proc 1964; 23 : 705-12.

17. Mahalanabis D, Choudhuri AB, Bagchi NG, et al. Oral fluid therapy of cholera among Bangladesh refugees. Johns Hopkins Med J 1973; 132 : 197-205.

18. Bernier JJ. Gastroentérologie. Paris : Flammarion Médecine-Sciences, 1984 : 1638.

19. Butler DG, Gall DG, Kelly MH, Hamilton JR. Transmissible gastroenteritis: mechanisms responsible for diarrhea in an acute viral enteritis in piglets. 7 Clin Invest 1974; 53: 1335-42.

20. Mahalanabis 'D, Patra FC. In search of a super oral rehydration solution: can optimum use of organic solute mediated sodium absorption lead to the development of an absorption promoting drug? J Diarrhoeal Dis Res 1983; 1: 76-81.

21. Patra FC, Mahalanabis D, Jalan KN, Sen A, Banerjec P. Is oral rice electrolyte solution superior to glucose electrolyte solution in infantile diarrhoca? Arch Dis Child 1982; 52: 910-2. clinique orientées par des constatations physiopathologiques.

Il y a 25 ans, à partir des principes de la thermodynamique irréversible [1o], deux lois physiologiques ont été formulées, deux qui sont directement à l'origine du traitement de la déshydratation par diarrhée aiguë : tout d'abord, à travers un épithélium comme celui de la paroi de l'intestin grêle, les mouvements d'absorption et de sécrétion de l'eau suivent passivement ceux des électrolytes et en particulier ceux du sodium [I I]; ensuite, les mouvements d'absorption du sodium sont liés à ceux du glucose par un système de cotransport au niveau de la membrane luminale de l'entérocyte [12]. A la même époque,
Finkelstein purifiait la toxine cholérique responsable de la diarrhée provoquée par le vibrion cholérique [13], permettant ainsi la reproduction expérimentale du choléra.

C'est de l'ensemble de ces résultats qu'est né le schéma thérapeutique actuel de la déshydratation. Il peut être résumé ainsi $[4,14]$ : en dehors de toute maladie, l'intestin est le siège d'une intense recirculation d'eau; entre les repas, l'eau provenant des sécrétions digestives de base (sécrétions salivaire, stomacale, biliaire, pancréatique et intestinale) entre dans la lumière intestinale, et est réabsorbée en suivant l'absorption active du sodium (figure 3). Au moment du repas, une grande quantité d'eau entre

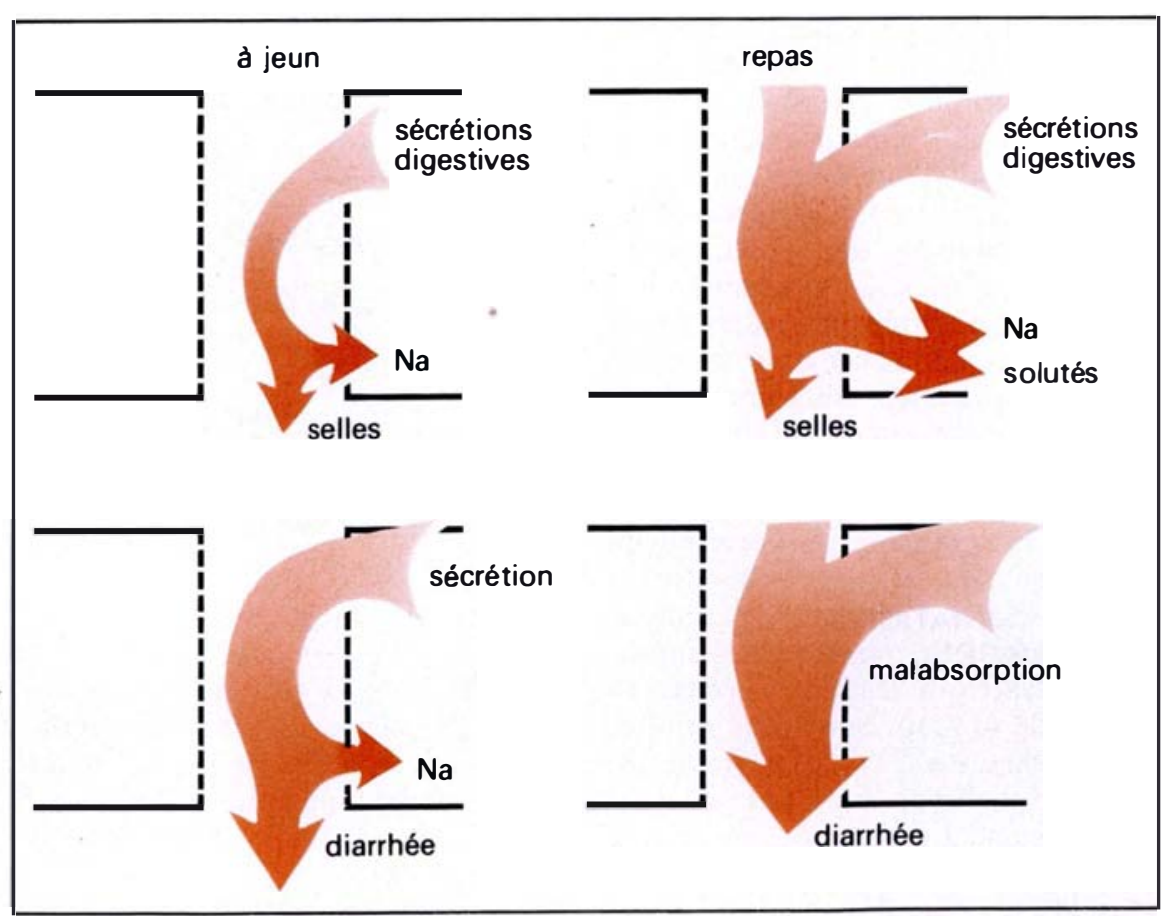

Figure 3. Le cycle entérosystémique de l'eau. A jeun (en haut à gauche) l'intestin réabsorbe l'eau venant des sécrétions digestives par un mécanisme faisant intervenir la réabsorption active du $\mathrm{Na}$. Au moment des repas (en haut à droite), la réabsorption du Na est stimulée par la présence des solutés dans la lumière intestinale. La diarrhée peut être due à une sécrétion (en bas à gauche) dépassant les capacités de réabsorption ou à une malabsorption (en bas à droite) (I4). 
dans la lumière intestinale du fait du volume du repas et plus encore de l'augmentation du volume des sécrétions digestives. La stimulation de la réabsorption de l'eau est due à une stimulation de la réabsorption du sodium, elle-même stimulée par la présence de glucose dans la lumière intestinale. Ainsi, physiologiquement, il existe un cycle entérosystémique de l'eau dont l'importance quantitative précise ne peut être donnée avec certitude du fait de l'incertitude sur la recirculation de l'eau au niveau de l'épithélium intestinal. Il est vraisemblable que chez le nourrisson, le volume réabsorbé représente plusieurs fois le volume total de l'eau du corps.

En altérant le cycle entérosystémique de l'eau, la diarrhée aiguë provoque rapidement une déshydratation grave. Plus précisément, la toxine cholérique stimule la sécrétion intestinale d'électrolytes qui entraîne à son tour une sécrétion d'eau. Lorsque la quantité sécrétée dépasse les capacités maximum de réabsorption par l'intestin grêle et le colon, la diarrhée se manifeste associée à la déshydratation. Fait fondamental, le système de réabsorption du sodium peut encore être stimulé par le glucose. C'est ainsi que l'on interprète l'effet thérapeutique du glucose sur la déshydratation provoquée par le vibrion cholérique

\begin{tabular}{|l|c|}
\hline \multicolumn{2}{|c|}{ Tableau III } \\
CONCENTRATION MOLAIRE \\
DES CONSTITUANTS \\
DE LA SOLUTION DE \\
RÉHYDRATATION ORALE [4]. \\
\hline constituant & $\mathrm{mmol} /$ litre d'eau \\
\hline sodium & 90 \\
potassium & 20 \\
chlorure & 80 \\
citrate & 10 \\
glucose & 111 \\
\hline
\end{tabular}

$m / s n^{0} 7$ novembre 85
$[15,16]$. Pour bien comprendre l'importance de cette découverte, il faut la replacer dans son contexte historique et géographique. Il y a 25 ans, le seul traitement efficace de la déshydratation aiguë consistait en une perfusion intraveineuse de solutés stériles d'électrolytes en milieu hospitalier. L'introduction de la réhydratation par voie orale laissait entrevoir une révolution dans la manière d'aborder ce traitement. La solution de réhydratation est préparée au moment de l'emploi par dilution d'un sachet d'électrolytes et de glucose dans un litre d'eau propre (tableau III). D'une grande simplicité, ce traitement a été immédiatement utilisé avec succès pour traiter l'épidémie de choléra qui s'est déclarée pendant la guerre du Bangladesh [ $\mathrm{r}$ 7]. Il est maintenant recommandé de préférence à la perfusion intraveineuse en Europe et en Amérique du Nord [18].

Pour mieux comprendre l'impact de ce traitement, il faut compléter le schéma initial par des informations plus récentes.

\section{Etiologie infectieuse}

Tout d'abord, il est maintenant bien établi que les diarrhées aiguës de l'enfant sont d'origine infectieuse [ $\mathrm{I}$ ]. Certains agents infectieux stimulent la sécrétion intestinale par l'intermédiaire de toxines, dont les mieux connues sont la toxine cholérique et les toxines LT et $\mathrm{ST}$ des $\mathrm{E}$. Coli entérotoxinogènes (ou ETEC), ou par l'intermédiaire de médiateurs sécrétagogues libérés par l'Entamoeba Histolytica. Par contre, d'autres agents ne stimulent pas la sécrétion intestinale mais diminuent la réabsorption. C'est le cas du rotavirus [I9]. Dans l'un et l'autre cas, la diarrhée se manifeste par une perte d'eau et d'électrolytes avec un risque de déshydratation. Fait remarquable, la réhydradation par voie orale est efficace cliniquement dans toutes ces diarrhées. D'autres bactéries provoquent la diarrhée par pénétration dans l'épithélium, souvent au niveau du colon; il s'agit surtout de souches d'E. Coli, ou de Shigelles ou de Salmonelles. Le tableau clinique est alors le plus souvent celui d'une diarrhée dysentérique. Dans ce cas, la réhydratation par voie orale n'a qu'un rôle d'appoint.

\section{Une meilleure solution de réhydratation?}

Le deuxième complément d'information porte sur les substrats qui stimulent l'absorption du sodium, par le système de cotransport au niveau de la membrane luminale, par entrainement avec les flux d'eau ou par d'autres mécanismes plus indirects encore. I.e fait important est que ces substrats viennent de l'alimentation. Ainsi, l'absorption est stimulée par les monosaccharides, glucose et galactose, mais pas par le fructose ou les disaccharides avant leur hydrolyse. Pour les protides, l'intensité de l'absorption dépend en partie de la nature de l'acide aminé ou du di ou tripeptide. Les acides gras à chaîne courte peuvent également stimuler l'absorption [I]. Pour renforcer l'efficacité de la solution de réhydratation, plusieurs techniques ont été proposées : (a) augmenter la concentration de glucose au-delà de I $10 \mathrm{mmol} / \mathrm{l}$; cela n'a fait qu'aggraver la diarrhée; (b) remplacer le glucose par un substrat plus facilement disponible et de même efficacité. A cet égard, le saccharose est un bon substitut du glucose; (c) ajouter des acides aminés ou des maltodextrines pour utiliser en même temps tous les moyens de stimuler l'absorption et ainsi réaliser une "super" solution de réhydratation [20]; les études cliniques sont en cours mais les premiers résultats sont encourageants. L'utilisation de diètes semi-élémentaires est limitée par leur disponibilité et leur prix de revient, pour un avantage qui n'est pas encore évalué cliniquement [I]; (d) utiliser des céréales disponibles localement dans la solution d'électrolytes [2I]. L'idée est que les céréales contiennent de l'amidon et des protéines qui sont hydrolysés dans la lumière intestinale sous forme de glucose, d'acides aminés et de petits peptides qui stimulent l'absorption de l'eau. L'avantage de cette approche tient au fait que ces solutions apporteraient plus de calories sans charge osmotique, du fait de la nature polymérique des substrats. Il faut cependant bien 


\section{RÉFÉRENCES}

22. Ficld $M$, Fromm D, Al-Awqati $Q$ Greenough WB. Effect of cholera enterotoxin on ion transport across isolated ileal mucosa. $7 \mathrm{Clin}$ Invest 1 972; 5 1: 796-804

23. Hautefeuille $M$, Peyrot M, Grasset E, Desjeux JF. Les antisécrétoires de la muqueuse intestinale. Vers un traitement pharmacologique des diarrhées aiguës. Gastroenterol Clin Biol 1984; 8: $436-43$.

24. Grasset E, Pinto M, Dussaulx E, Zweibaum A, Desjeux JF. Epithelial properties of human colonic carcinoma cell line Caco-2: electrical parameters. Am 7 Physiol 1984; 247 (Cell Physiol 16): C260-C67.

25. Tai YH, Feser J, Marnane WG, Desjeux JF. Antisecretory effects of berberine in rat ileum. Am J Physiol 1981; 24I (Gastrointest Liver Physiol 4): G253-G58.

26. Isely RB. Relating improvements in water supply and sanitation to nutritional status: the issue of using anthropometry as an evaluation measure. Food and Nutrition Bulletin 1984; 5: 42-5I.

27. Feachem RG, Hogan RC, Merson $M H$ Diarrhocal disease control: reviews of potential interventions. Bull $W I J O$ 1983; 61: 637-40.

28. A positive effect on the nutrition of Phillipine children of an oral glucose-electrolyte solution given at home for the treatment of diarrhea. Report of a field trial by an international study group. Bull $W H O$ I $977 ; 55: 87-94$.

29. Santosham M. Nutritional benefits related to oral therapy. In: Bcllanti JA, ed. Acute diarrhea; its nutritional consequences in children. New York: Raven Press, 1983: 193-7.

30. Nalin Dr. Nutritional benefits related to oral therapy. In: Holmc T, Holmgren J, Merson MH, Mollby $\mathrm{R}$, eds. Acute enteric infection in children. New prospects for treatment and prevention. Amsterdam: Elsevier North Biochemical Press, 1981: i 85-91.

31. Ben Mansour A, Achour A, Chabchoub S, Nagati K, Desjeux JF. Fffets comparés de la solution de l'OMS et d'une solution simplifiée chez le nourrisson présentant une diarrhée aiguë. Ann Pédiatr (Paris) 1985; 32:315-20. réaliser que la solution complète de réhydratation additionnée de céréales ne doit être conçue que temporairement pour augmenter l'efficacité de la réhydratation et non comme une source d'énergie; sinon, elle risque de retarder la reprise de l'alimentation, y compris de l'allaitement maternel dès la fin de la réhydratation. De plus, il existe un risque de surcharge en électrolytes après la phase initiale de réhydratation, en particulier dans les régions où les céréales constituent une part importante de l'alimentation familiale.

A l'évidence, il reste là tout un domaine de recherche pour améliorer l'absorption de l'eau en modifiant la composition des solutions, dans des conditions d'utilisation souvent très rudimentaires.

\section{Sécrétion intestinale : mécanismes cellulaires}

Le troisième domaine en plein développement concerne les mécanismes de la sécrétion intestinale. Sans entrer dans les détails, il faut noter que la notion même de sécrétion intestinale n'a pu s'imposer que parce qu'elle était nécessaire à l'explication de la diarrhée induite par la toxine cholérique [22]. Cette sécrétion d'électrolytes, essentiellement de chlore, est un processus actif régulé par les médiateurs chimiques intracellulaires communs aux autres cellules: l'AMP cyclique, le GMP cyclique, le calcium libre intracellulaire et les métabolites de l'acide arachidonique [23]. L'explication du mécanisme intime au niveau cellulaire a été limité jusqu'à présent par la complexité de l'épithélium. La sélection de cellules intestinales d'origine humaine en culture montrant des propriétés sécrétrices, devrait permettre dans un proche avenir de connaitre les mécanismes cellulaires de la sécrétion [24]. Ceci est important pour définir des agents antisécrétoires; il faut toutefois noter que pour l'instant, ces agents n'ont pas prouvé leur efficacité dans le traitement clinique de la diarrhée. A l'inverse, des médicaments utilisés en médecine traditionnelle, comme la berbérine qui est un alcaloide du Berberis Aristata, ou la caséine méthylée, se sont montrés, in vitro, de puissants antisécrétoires [25]. En résumé, la réhydratation par voie orale est maintenant un traitement simple et efficace de la déshy-

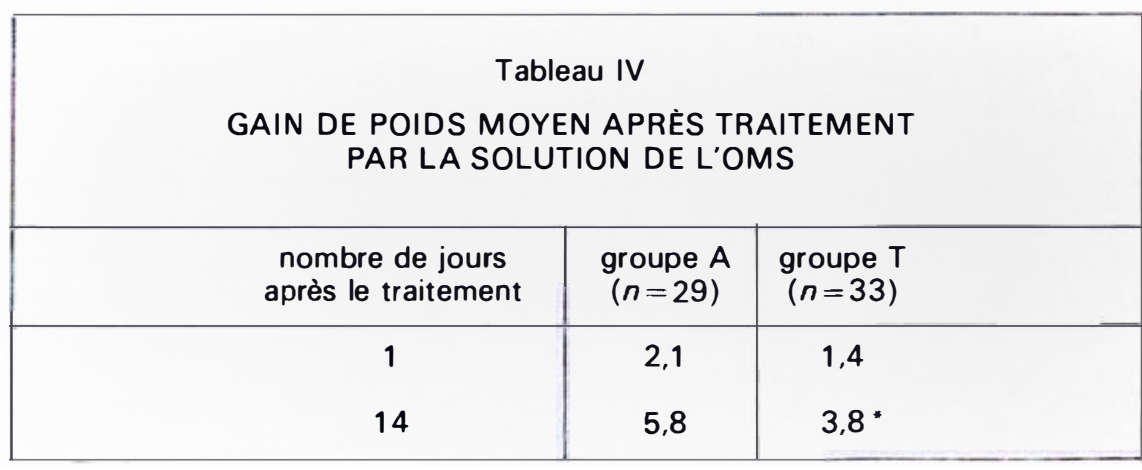

Le groupe A a été traité par la solution de l'OMS donnée de manière appropriée; le groupe témoin (groupe $T$ ) a ręu les boissons et les aliments habituels. Les résultats sont exprimés en $\%$ du poids initial /29/.

* Différence significative entre les deux groupes $(p<0,05)$. 
dratation par diarrhée, fondée sur des bases physiopathologiques précises; les études cliniques indiquent qu'elle s'applique à toutes les étiologies, à tous les âges et à toutes les régions; son efficacité est cependant soumise au respect d'une technique précise d'utilisation [4].

\section{Réhydratation et état nutritionnel}

Après avoir montré le rôle des diarrhées aiguës infectieuses dans la malnutrition et après avoir mis au point un traitement efficace de la diarrhée, il semblait logique d'essayer de vérifier les conséquences nutritionnelles de la réhydratation par voie orale. Cette question peut se poser également pour toutes les mesures préventives ou curatives de la diarrhée, telles que l'introduction de l'eau potable, le maintien ou le développement de l'allaitement maternel, l'éducation sanitaire, la surveillance de la croissance, les vaccinations contre les agents de la diarrhée et contre la rougeole, maladie souvent associée à la diarrhée $[26,27]$. Bien que les résultats ne soient pas toujours faciles à analyser statistiquement, une amélioration de l'état nutritionnel a été notée dès les premières années de l'utilisation systématique de la réhydratation par voie orale [28]. Dans l'ensemble, les études prospectives ont comparé 2 groupes d'enfants dont l'un recevait une réhydratation vigoureuse par une solution de glucose et d'électrolytes sans interruption de l'alimentation, et l'autre, le traitement en usage localement, y compris la prise de boissons et d'aliments. A titre d'exemple, le tableau IV montre les résultats d'une étude de ce type à Panama [29]. Des résultats allant également dans le sens d'une amélioration nutritionnelle ont été obtenus en Extrême-Orient, au MoyenOrient et en Amérique Latine. L'effet bénéfique de ce traitement n'a pu cependant être mis en évidence en Gambie.

Même si la réalité et l'amplitude de l'effet est difficile à cerner, il semble très important d'essayer d'en comprendre les mécanismes. La première constatation est que l'effet bénéfique ne s'observe, semble-t-il, que si la technique de la réhydratation est en accord avec ces quatre objectifs [4] : (a) compensation du déficit en eau et en électrolytes présent au début du traitement, (b) compensation des pertes anormales dues à la persistance de la diarrhée et des vomissements, (c) couverture des besoins de base en eau pendant la durée du traitement, (d) initiation de la récupération nutritionnelle. En d'autres termes, il ne faut pas considérer la composition de la solution de réhydratàtion indépendamment de la technique de réhydratation ellemême. Le deuxième commentaire porte sur les mécanismes de l'effet trophique de la réhydratation orale. Ils peuvent être de l'ordre du comportement et de l'ordre de la physiologie [30] : le nourrisson mange plus, plus tôt et mieux grâce aux instructions, à la motivation et à l'encouragement de la mère et du personnel soignant; la solution de réhydratation faciliterait l'alimentation et la récupération nutritionnelle précoces par le rétablissement rapide de la dynamique circulatoire et par l'apport de potassium. Autrement dit, même si une diarrhée aiguë banale chez l'enfant sain entraîne des désordres métaboliques sur plusieurs jours, il est vraisemblable qu'une réhydratation lente ou incomplète, associée à un retard de l'alimentation, serait néfaste et ne ferait que prolonger la maladie. Le troisième commentaire porte sur ce qui accompagne le geste thérapeutique. Tous les auteurs s'accordent pour signaler que l'effet de la réhydratation par voie orale est fortement lié au temps passé par l'agent médical à expliquer le mode d'emploi de ce traitement [3I]. Enfin, il est important de noter que le traitement a généralement un effet bénéfique à long terme en réduisant la malnutrition qui aggrave l'évolution de la diarrhée aiguë

En conclusion, cet article n'avait pas comme objectif de décrire les moyens de lutter contre la diarrhée ou la malnutrition. Il existe de nombreux documents à ce sujet. Bien plutôt il souhaitait montrer les multiples facettes des approches technologiques utilisées pour aborder un problème nutritionnel de première urgence

\section{Summary}

Malnutrition in children is the consequence of numerous factors, including infectious diseases. Among these, acute diarrhea is a leading cause of death in the world, and it is becoming the most prevalent disease. It is an essential factor in the initiation, development and aggravation of malnutrition. The curative treatment of acute dehydration with an oral electrolyte and glucose solution has been derived from basic physiological concepts in accordance with irreversible thermodynamics. The utilization of this treatment is the basis of a worldwide strategy which contributes to decrease malnutrition in children.

\section{TIRES A PART}

J.-F. Desjeux : Inserm U 83, CHU Villemin, 10, avenue de Verdun, 75010 Paris. 